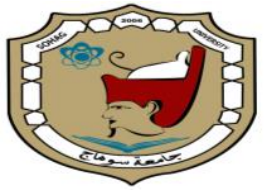

Sohag University
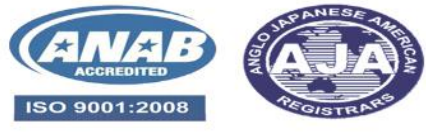

Sohag Medical Journal

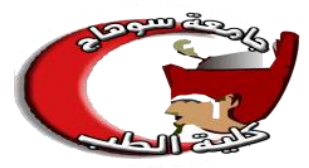

Faculty of Medicine

\title{
The role of the cerebellum in the pathogenesis of essential tremor
}

\section{Gharib Fawi, Hazem K Elhewag, Mohamed Nasreldin Thabit, Ahmed Ezzat Amin}

Department of Neurology and Psychological medicine, Faculty of Medicine, Sohag

University

\begin{abstract}
Abnormal activity in a neuronal network in which the cerebellum is included is coherent with continuous tremor oscillation. Within the cortico-Olivo-cerebella-thalamic circuit, the inferior olive could be an oscillator by having pacemaker properties. Despite that, the olivary pacemaker hypothesis faces many contradicting findings against the concept of a single oscillator. The attention has changed from the interactions between the elements and structures of the neural network in which the oscillators are contained to concentrate on its features. These features include the intensity and direction of the connection. Deficiency in the glutamatergic system in ET may be a better explanation for abnormal cerebellar function in ET. A reduction in dentate GABA receptor levels could be a basic defect in this disease. This may be explained by the reduced production of GABA from Purkinje cells at the postsynaptic neurons. This results in the release of deep cerebellar nuclei from previous inhibition. The correlation between genetic abnormalities and cerebellar dysfunction may explain this in the future.
\end{abstract}

Keywords: essential tremor, cerebellum, olivary pacemaker, GABA.

\section{Introduction}

There is an agreement that central mechanisms have a role in essential tremors (ET). The neuronal activity in a network consisting of the frontal cortex, cerebellum, thalamus, and the olivary region, has been revealed to be coherent with continuous tremor oscillation (using electromyography, (EMG)) [1, 2]. ET cases were found to have higher cerebellar activity when compared to simulated tremor, according to functional magnetic resonance imaging (fMRI) studies ${ }^{[3]}$. ET patients have altered connections in the cerebellum-dentate-thalamic tract, according to other fMRI studies with emph- asis on the connectivity between different regions ${ }^{[4]}$.

These findings imply that ET is linked to an increase in tremor-related activity in many cerebellar circuit theories that could explain the malfunctioning of the cortico-Olivo-cerebellum-thalamic network in ET includes:

\section{1-The hypothesis of the olivary pac- emaker:}

The hyperpolarization of some neuronal membranes causes them to oscillate at a set frequency independently ${ }^{[5]}$. Within the cortico-Olivo-cerebella-thalamic circuit, the inferior olive, which possesses these pacemaker qualities, could be an 
oscillator $^{[6] .}$ It demonstrates elevated expression of EAAT2, a key glutamate reuptake transporter linked to the ET phenotype in genome-wide association investigations ${ }^{[7]}$. The fact that rhythmic stimulation can entice ET across various areas (thalamus and cerebellum) contradicts the concept that there is a single oscillator (or pacemaker) ${ }^{[8]}$. Multiple, geographically isolated tremor clusters within the posterior division of the ventrolateral thalamus (VL) are capable of driving the tremor, according to investigations employing deep brain stimulation (DBS) electrodes in individuals with ET and tremor-dominant Parkinson's disease (PD), ${ }^{[9]}$. This is another evidence against the concept of a single oscillator.

These discoveries have redirected focus to network features such as the strength and direction of connectivity between these regions, which consider the neural network containing these oscillators, not on the relations between the network's elements and structures ${ }^{[10]}$. Although both voluntary and involuntary tremors are caused by the same circuit ${ }^{[1]}$, the involuntary tremors have thalamocortical connections which are different in being in both directions $\left.{ }^{[11}\right]$.

The data mentioned previously point to a changing pattern of cooperation among all tremor network members, with the exact network composition altering over time ${ }^{[12]}$. So, all the components of networks can entrain each other in a dynamic way and behave as oscillators. A previous question was why some ET improves after cerebellar stroke could be answered based on that ${ }^{[13]}$. but returns after ipsilateral cerebellectomy in others ${ }^{[14]}$. It also corresponds with the finding that ET can be removed by lesions in multiple locations within the cerebello- thalamocortical circuit, arguing against a single oscillator ${ }^{[13]}$.

\section{2-The GABA Hypothesis:}

The strongest evidence about ET neurochemistry is related to the Gammaaminobutyric acid (GABA), GABAergic, and glutamatergic systems, with a piece of weaker evidence for adenosine, dopaminergic, and adrenergic systems, while there is insufficient data about other neurotransmitter systems ${ }^{[15]}$. Purkinje cell loss is one of the morphological alterations seen in ET patients ${ }^{[16]}$, and another set of patients had enhanced Purkinje cell recurrent collateral development ${ }^{[17]}$. Purkinje cell loss reduces inhibitory GABAergic cerebellar corticofugal output, while Purkinje cell recurrent collateral development increases inhibition of neighboring Purkinje cells, lowering inhibitory GABAergic output [16]. Increased basket cell axonal plexus hypertrophy also leads to increased Purkinje cell inhibition, resulting in diminished Purkinje cell inhibitory GABAergic output ${ }^{[18]}$. The efficacy of primidone, topiramate, gabapentin, and ethanol in ET provides further evidence for GABA's role in ET as these drugs enhance GABA [19]. On the other hand, harmaline is thought to elicit tremor by blocking GABA-A receptors, causing increased electrical coupling of cerebellar afferents in the inferior olive ${ }^{[20]}$ Also, a postural tremor in lab animals was elicited by blocking GABAergic transmission and ET-like postural and kinetic tremor was observed in GABA-A receptor 1 deletion in mice ${ }^{[21]}$.

GABA receptor binding was discovered to be changed in nuclear imaging tests in ET. Positron emission tomography (PET) was used in a study and was found that enhanced 11Cflumazenil binding to GABA-A receptors in the ventrolateral thalamus, the dentate nucleus of 
the cerebellum, and the premotor cortex in eight ET patients was higher than in healthy controls ${ }^{[22]}$. In an ET postmortem research comparing between ET cases, controls, and PD patients, levels of GABA-A and GABA-B receptors in the dentate nucleus of the cerebellum were shown to be lowest in ET ${ }^{[23]}$.

According to the previous findings, a reduction in dentate GABA receptor levels could be a basic defect in this illness, by the reduced production of GABA from Purkinje cells at the postsynaptic neurons. This in turn results in the release of deep cerebellar nuclei from previous inhibition. ${ }^{[23]}$. This cause the deep cerebellar nuclei neurons to be overactive and potentially causing tremor by traveling up the cerebellothalamocortical circuit.

Despite these findings, which point to GABA's role in ET, no genetic factors have been discovered to explain these GABAergic abnormalities in ET, and no evidence for a link between GABA receptor and transporter polymorphisms and ET ${ }^{[24,25]}$.

\section{Conclusion}

The cerebellar dysfunction in ET is a well-established feature in ET. The olivary pacemaker theory failed to explain all findings in ET cases The GABA hypothesis is more and more gaining trust in explaining the abnormal cerebellar function in ET. Despite that further studies are needed searching for genetic factors that could explain these abnormal findings

\section{References}

1. Schnitzler, A., et al., Synchronized brain network associated with essential tremor as revealed by magnetoencephalography. Movement disorders: official journal of the Movement Disorder Society, 2009. 24(11): p. 1629-1635.

2. Muthuraman, M., et al., Essential and aging-related tremor: Differences of central control. Movement Disorders, 2015. 30(12): p. 1673-1680.

3. Broersma, M., et al., Bilateral cerebellar activation in unilaterally challenged essential tremor. Neuroimage Clin, 2016. 11: p. 1-9.

4. Gallea, C., et al., Intrinsic signature of essential tremor in the cerebellum-frontal network. Brain, 2015. 138(10): p. 29202933.

5. Llinás, R.R., The intrinsic electrophysiological properties of mammalian neurons: insights into central nervous system function. Science, 1988. 242(4886): p. 1654-1664.

6. Helmich, R.C., et al., The pathophysiology of essential tremor and Parkinson's tremor. Curr Neurol Neurosci Rep, 2013. 13(9): p. 378.

7. There, S., et al., Polymorphisms in the glial glutamate transporter SLC1A2 are associated with essential tremor. Neurology, 2012. 79(3): p. 243-248.

8. Carignan, H., et al., The nature of tremor circuits in parkinsonian and essential tremor. Brain, 2014. 137(12): p. 32233234.

9. Pedrosa, D.J., et al., Essential tremor and tremor in Parkinson's disease are associated with distinct 'tremor clusters' in the ventral thalamus. Experimental neurology, 2012. 237(2): p. 435-443.

10.Helmich, R.C., et al., The pathophysiology of essential tremor and Parkinson's tremor. Current neurology and neuroscience reports, 2013. 13(9): p. 378.

11.Muthuraman, M., et al., Oscillating central motor networks in pathological tremors and voluntary movements. What 
makes the difference? Neuroimage, 2012. 60(2): p. 1331-1339.

12.Raethjen, J. and G. Deuschl, The oscillating central network of essential tremor. Clinical neurophysiology, 2012. 123(1): p. 61-64.

13.Dupuis, M.J.M., et al., Disappearance of essential tremor after stroke. Movement disorders, 2010. 25(16): p. 2884-2887.

14. Chahine, L.M. and D. Ghosh, Essential tremor after ipsilateral cerebellar hemispherectomy: support for the thalamus as the central oscillator. Journal of child neurology, 2009. 24(7): p. 861864.

15.Jiménez-Jiménez, F.J., et al., An Update on the Neurochemistry of Essential Tremor. Current medicinal chemistry, 2019.

16.Louis, E.D., et al., Neuropathological changes in essential tremor: 33 cases compared with 21 controls. Brain, 2007. 130(Pt 12): p. 3297-307.

17.Babij, R., et al., Purkinje cell axonal anatomy: quantifying morphometric changes in essential tremor versus control brains. Brain, 2013. 136(10): p. 3051-3061.

18.Kuo, S.-H., et al., Lingo-1 expression is increased in essential tremor cerebellum and is present in the basket cell pinceau. Acta neuropathologica, 2013. 125(6): p. 879-889.
19.Deuschl, G., et al., Treatment of patients with essential tremor. The Lancet Neurology, 2011. 10(2): p. 148161.

20.Stratton, S. and J. Lorden, Effect of harmaline on cells of the inferior olive in the absence of tremor: differential response of genetically dystonic and harmaline-tolerant rats. Neuroscience, 1991. 41(2-3): p. 543-549.

21.Kralic, J.E., et al., Genetic essential tremor in $\gamma$-aminobutyric acid A receptor $\alpha 1$ subunit knockout mice. The Journal of clinical investigation, 2005. 115(3): p. 774-779.

22. Gironell, A., et al., Gaba and serotonin molecular neuroimaging in essential tremor: a clinical correlation study. Parkinsonism \& related disorders, 2012. 18(7): p. 876-880.

23.Paris-Robidas, S., et al., Defective dentate nucleus GABA receptors in essential tremor. Brain, 2012. 135(1): p. 105-116.

24.García-Martín, E., et al., Gammaaminobutyric acid GABRA4, GABRE, and GABRQ receptor polymorphisms and risk for essential tremor. Pharmacogenetics and genomics, 2011. 21(7): p. 436-439.

25.Their, S., et al., GABAA receptor-and GABA transporter polymorphisms and risk for essential tremor. European journal of neurology, 2011. 18(8): p. 1098-1100. 